\title{
Healthcare Overuse: A Literature Review and Taxonomy Proposal
}

\author{
Eva Koeller ${ }^{1}$, Colleen Barclay ${ }^{2}$, Tina L. Velasquez ${ }^{1}$, Russell Harris ${ }^{2}$, Melissa R. Partin ${ }^{1}$, Elisheva R. Danan ${ }^{1,3}$, \\ Nancy Greer ${ }^{1}$ and Timothy J. Wilt ${ }^{1,3}$ \\ 1. Center for Chronic Disease Outcomes Research and the Minneapolis VA High Value Care Initiative, Minneapolis VA Health Care \\ System, Minneapolis MN 55417, USA \\ 2. Sheps Center for Health Services Research, University of North Carolina at Chapel Hill, Chapel Hill NC 27599, USA \\ 3. Department of Medicine, Section of General Internal Medicine, University of Minnesota, Minneapolis MN 55417, USA
}

\begin{abstract}
Healthcare overuse is the provision of care in which the benefits do not justify the harms and/or costs. Overuse literature is poorly categorized thus impeding research, practice, and policy to reduce overuse and improve healthcare quality. We developed an overuse taxonomy and searched for and reviewed the healthcare overuse literature in an attempt to better understand and categorize research on overuse practice and patterns. We found that more than two-thirds of articles were observational $(70 \%)$, the most prevalent purpose of overuse was treatment/secondary prevention (69\%), the most common type of overuse was overtreatment (73\%), drivers of and methods to reduce overuse were each discussed in about $40 \%$ of abstracts, and the most frequently mentioned clinical area was pharmacy. A high volume of overuse literature exists. However, the majority of overuse research is observational, descriptive, and focuses on overtreatment and overprescribing rather than methods to reduce overuse. Some overuse is not labelled as such. Our taxonomy adequately organized the existing literature and identified areas where additional research efforts are most needed. A common taxonomy, such as ours, could help researchers categorize their work, assist clinicians and policymakers in identifying and implementing findings, and guide future research to improve healthcare quality.
\end{abstract}

Key words: Healthcare overuse, overtreatment, overuse, healthcare utilization.

Disclaimer: The views expressed in this article are those of the authors and do not necessarily reflect the position or policy of the Department of Veterans Affairs or the United States government.

\section{Introduction}

Healthcare overuse occurs when patients receive care they don't need, that doesn't improve their health, or that exposes them to costs, harms, and risks that are excessive when compared to the benefits or to alternative options for care [1]. An estimated $30 \%$ of the United States' healthcare dollars are spent on care that fits this definition [2]. Increased healthcare use

Corresponding author: Timothy J. Wilt, MD, MPH, professor, research fields: disease prevention, health promotion, chronic disease management, cancer screening, health services research, epidemiology and adults. and overuse have been associated with worse outcomes, higher mortality, decreased patient satisfaction, and higher costs [3]. Reducing healthcare overuse will improve clinical outcomes and patient experiences, reduce costs, and make more resources available to provide high-value care to more individuals.

Unfortunately, conducting research in this area has been difficult, in part because few studies have assessed overuse and the existing literature is poorly categorized. A 2012 systematic review performed a search over 21 years (1978-2009) and identified only 172 articles measuring overuse, most of which addressed antibiotic use for upper respiratory infections or one of several cardiac interventions [4]. The authors concluded that the limited overuse literature was due to the difficulty in measuring overuse and noted that the search was complicated by the lack of Medical 
Subject Heading (MeSH) terminology for overuse [4]. The current state of overuse literature is hindering research, practice, and policy to reduce overuse and improve healthcare quality.

We searched for and reviewed the healthcare overuse literature and developed a priori a taxonomy to categorize the identified literature according to: purpose of care, type of overuse, and state-of-the-research in order to develop a clearer understanding of the current state of practice and patterns related to overuse. Our goal was to gain insight into more effective ways to categorize the literature, assess the range of overuse literature and how well studies fit our proposed taxonomy, and describe a possible framework for future research to improve healthcare value by reducing overuse.

\section{Materials and Methods}

\subsection{Taxonomy Development}

We developed our taxonomy during iterative discussions with all of the contributing authors, which were informed by the individual authors' previous knowledge of and experience with overuse literature. We also conducted pilot-testing of our taxonomy during which we regularly adapted and refined our classifications to more closely reflect the healthcare overuse literature. We attempted to choose categories that would be informative and provide a clear picture of what work is being done and where research is lacking. The taxonomy used in this paper is the final result of this process and was applied to the final set of randomly selected articles. A literature search and review were done in part to see whether our taxonomy adequately organized the existing literature and whether there were studies that didn't fit our taxonomy or categories in which additional research was needed.

\subsection{Database Search}

We conducted a search of Ovid (Medline) from January 2000 through January 2015 for English language literature, of any study design, in adults. Our search strategy was based on that used by Kressin with modifications based on studies done by Morgan and Korenstein [3-5]. Search terms included: health services misuse, inappropriate utilization, overuse, overtreatment, and overdiagnosis. We limited our results to papers with available abstracts and the search terms in their titles. We defined overuse as the receipt of care which is unneeded, which doesn't improve health outcomes, or that may expose patients to excessive costs, harms, and risks as compared to the benefits or alternative options for care [1] and excluded papers that were not relevant to healthcare overuse; such as overuse injuries. We also excluded studies in women who were pregnant or breast feeding and dental or lab-based research. We included all article types. Three trained researchers independently reviewed, in duplicate, a 10 percent random sample of the resulting abstracts, selected using a random number generator. Researchers determined whether the abstracts met the inclusion criteria described above with disagreements resolved by discussion with all reviewers. Reviewers also classified any included articles based on our a priori taxonomy.

\subsection{Abstract Categorization}

Our taxonomy categorizes studies based on three factors: 1) purpose of care (primary prevention, diagnosis, treatment/secondary prevention, screening/monitoring, or other), 2) type of overuse (overtesting, overdiagnosis, overtreatment, or other), and 3) state-of-the-research (drivers of overuse, barriers-to-reducing overuse, methods to avoid overuse, or prevalence). We also noted the clinical area/specialty and study design. We developed $a$ priori definitions of these broad categories to classify articles based on the main themes identified in the abstract.

\subsubsection{Purpose of Care}

We defined primary prevention as preventing disease in apparently healthy people (e.g. prophylactic 
antibiotics) [6]. We defined diagnosis as the process of determining the nature or cause of maladies; including imaging for low back pain and biopsy for suspected cancer [7]. Treatment/secondary prevention included medications and procedures used to manage, improve, or cure a condition or prevent it from developing further [8]. Screening/monitoring included procedures done to look for the presence or track the progression of a disease [6, 8]. Although cancer screening is in some ways preventive we classified it as screening because in most cases the actual screening test (e.g. mammogram) does not itself prevent the condition. For screening colonoscopies and other screening or diagnostic procedures where the objective can be both detection and prevention we classified papers as screening unless the abstract indicated that the overuse was related specifically to prevention (e.g. intervening for low-grade cervical dysplasia as opposed to screening too frequently). Abstracts could be counted in multiple healthcare areas but we attempted to choose one category that fit best. We classified abstracts that did not fit into any of these categories as other.

\subsubsection{Type of Overuse}

We defined overtesting as the use of non-recommended tests or testing in inappropriate individuals or at inappropriate times [9]. This could include use of a recommended test in patients for whom the test is not indicated, for example too young or old, or testing more frequently than recommended. Additionally, it could include testing with a test that is more sensitive or has more harms or costs than the recommended test. We defined overdiagnosis as, "the diagnosis of conditions that will never cause symptoms or death" and overtreatment as, "treatment that includes overdiagnosed conditions..., that is futile, has minimal evidence of benefit for the specific indications, or is excessive (in complexity, duration, harms or cost) relative to alternative accepted standards" [3]. Abstracts were counted in as many categories as were relevant.

\subsubsection{State-of-the-research}

We defined a driver of overuse as any factor that increases the provision of low-value care. Abstracts designated as drivers included those that looked at the association between patient characteristics, providers, or other factors and overuse. Barriers-to-reducing overuse are factors that prevent doctors, patients, health systems or other health professionals from reducing overuse. Methods to reduce overuse includes strategies used to avoid or decrease overuse. The success of these strategies, as described by the abstract, was not taken into account and both experimental and conceptual discussion of strategies was allowed. Abstracts that did not contain any of these three topics were classified as only concerning prevalence of overuse. We marked as many topics as were relevant, except prevalence, which we chose only if no other topic was appropriate.

We also constructed very brief summaries of each abstract noting the clinical area and study design.

\section{Results}

The search resulted in 2,843 references, of which a random 10 percent sample $(n=283)$ of abstracts underwent dual-review and $131(46 \%)$ were deemed eligible and were categorized (Figs. 1 and 2, Table 1).

Inter-rater agreement for eligibility was good $($ Cohen's kappa $=0.74)$. Of the 131 eligible abstracts, $70 \%(\mathrm{n}=92)$ were observational studies. Only $4 \%$ $(\mathrm{n}=5)$ were randomized or controlled clinical trials and $3 \%(n=4)$ were systematic reviews. The remainder consisted of theory/conceptual papers, surveys, narrative reviews, modelling studies, qualitative studies, and case reports (Fig. 1). We found only one controlled trial testing an intervention to reduce overuse. This was a cluster randomized trial of an educational intervention to reduce inappropriate benzodiazepine prescriptions [10].

\subsection{Purpose of Care}

More than two-thirds $(69 \%, \mathrm{n}=91)$ of the included 


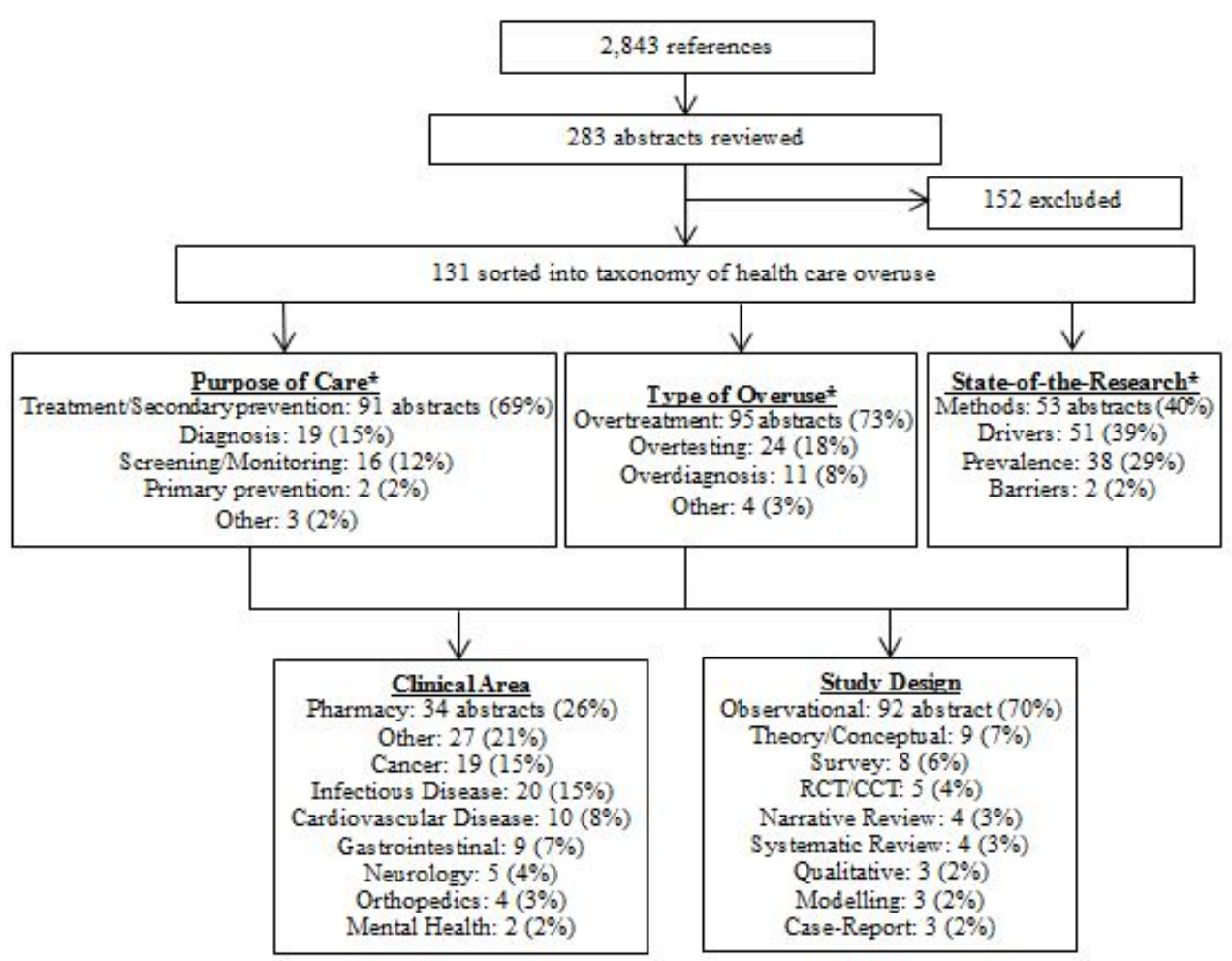

Fig. 1 Literature Flow *indicates that abstracts could have been counted in more than one category.



Fig. 2 Distribution of abstracts by purpose of care, type of overuse, and state-of-the-research. 
Table 1 Abstract count.

\begin{tabular}{|c|c|c|c|c|c|}
\hline \multirow[b]{2}{*}{ Purpose of Care } & \multirow[b]{2}{*}{ Type of Overuse } & \multicolumn{4}{|c|}{ Number of Abstracts } \\
\hline & & Drivers & $\begin{array}{l}\text { Barriers to } \\
\text { Reducing }\end{array}$ & $\begin{array}{l}\text { Methods to } \\
\text { Avoid/Reduce }\end{array}$ & Prevalence \\
\hline $\begin{array}{l}\text { Primary Prevention } \\
(2 \text { abstracts) }\end{array}$ & Overtreatment & 1 & & & 1 \\
\hline \multirow{3}{*}{$\begin{array}{l}\text { Diagnosis } \\
\text { (19 abstracts) }\end{array}$} & Overtesting & 2 & & 5 & 7 \\
\hline & Overdiagnosis & 2 & & 1 & 1 \\
\hline & Overtreatment & & & 1 & \\
\hline \multirow{2}{*}{$\begin{array}{l}\text { Treatment/Secondary } \\
\text { Prevention } \\
\text { (91 abstracts) }\end{array}$} & Overtreatment & 38 & 2 & 41 & 22 \\
\hline & Other & & & 1 & \\
\hline \multirow{3}{*}{$\begin{array}{l}\text { Monitoring/Screening } \\
\text { (16 abstracts) }\end{array}$} & Overtesting & 7 & & 1 & 3 \\
\hline & Overdiagnosis & 2 & & 1 & 4 \\
\hline & Overtreatment & 2 & & & \\
\hline Other (3 abstracts) & Other & & & 3 & \\
\hline
\end{tabular}

overuse abstracts evaluated treatments or secondary prevention, including articles on inappropriate use of medications and hospitalizations. This was followed by $15 \%(\mathrm{n}=19)$ on diagnosis, and $12 \%(\mathrm{n}=16)$ on screening/monitoring (Fig. 1). Only $2 \%(n=2)$ of the abstracts concerned interventions intended for primary prevention, the same proportion that did not fit into any predetermined category (other).

\subsection{Type of Overuse}

The most common type of overuse reported was overtreatment, which was the focus of $73 \%(n=95)$ of all included abstracts $(n=131)$. These abstracts included studies of fracture management, antimicrobial use, and inappropriate prescribing, among other topics [11-13]. The second most prevalent type of overuse was overtesting $(18 \%, \mathrm{n}=24)$, followed by overdiagnosis $(8 \%, \mathrm{n}=11)$, and other $(3 \%, \mathrm{n}=4)$

(Fig. 1). Several abstracts described more than one type of overuse and were counted multiple times. Overtreatment was the subject of abstracts concerning all four purposes of care and it accounted for 100\% $(n=2)$ of the primary prevention abstracts and $99 \%$ $(n=90)$ of the secondary prevention/treatment abstracts. Overtesting was mentioned in the context of diagnosis $(n=14)$ and screening/monitoring $(n=10)$. These included abstracts on the use of magnetic resonance imaging, mammography, and $\mathrm{C}$. difficile [14-16]. Overdiagnosis was the subject of abstracts concerning screening/monitoring $(\mathrm{n}=7)$ and diagnosis $(\mathrm{n}=4)$, including research on breast, prostate, and lung cancer, as well as malaria and bipolar disorder $[17,18]$.

\subsection{State-of-the-Research}

Twenty nine percent $(n=38)$ of the 131 included abstracts described only overuse prevalence. These abstracts describe studies with topics such as the inappropriate use of medications and nuclear stress tests $[19,20]$. For all other categories, abstracts could be counted multiple times. We found that $39 \%(n=51)$ described potential drivers of overuse and $40 \%(n=53)$ referred to potential methods to reduce overuse, most only conceptually (Fig. 1$)$. Only $2 \%(n=2)$ of the abstracts mentioned potential barriers-to-reducing overuse. This pattern persisted when results were analyzed by purpose of care. The most commonly reported driver of overuse was patient characteristics (e.g. race, gender, age), which was reported in 29 of 51 $(56 \%)$ driver abstracts, followed by the clinician (13 abstracts), the healthcare system (nine abstracts), guidelines/tools (four abstracts), and education or lack thereof (one abstract) (Fig. 3).

Although the most frequently mentioned driver of overuse was patient characteristics, the method most commonly suggested to reduce overuse was, change to or implementation of, guidelines and other tools; reported in 16 out of 53 methods abstracts. This was followed by interventions targeted towards the 


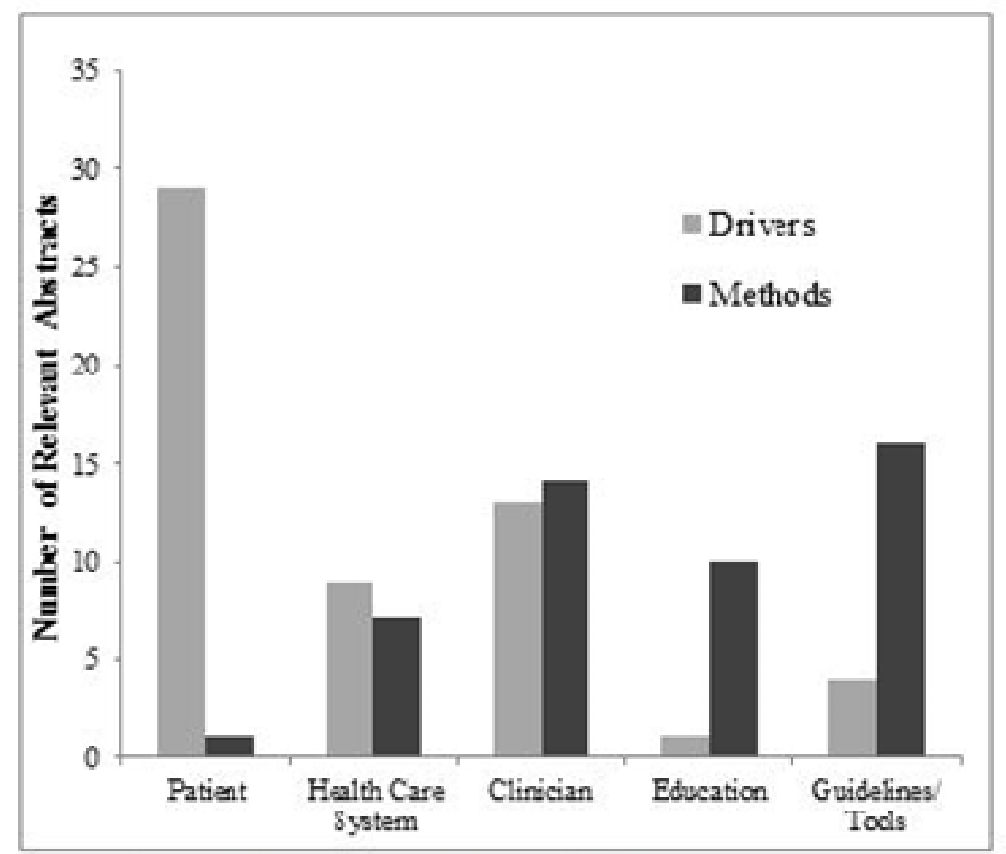

Fig. 3 Drivers of and methods to reduce overuse by target.

clinician (14 abstracts), education of the patient, practitioner or other (10 abstracts), changes to the healthcare system (seven abstracts), and the patient (one abstract) (Fig. 3).

\subsection{Overuse by Clinical Area}

Along with categorizing abstracts according to our taxonomy, we noted the different clinical areas/health conditions and medical specialties represented in the overuse literature. The most common clinical area was pharmacy, which was the subject of $26 \%(\mathrm{n}=34)$ of included abstracts $(n=131)$ (Fig. 1). These abstracts primarily described the prescription of inappropriate medications in the elderly. Fifteen percent $(n=19)$ of included abstracts were on cancer and $15 \%(\mathrm{n}=20)$ were on infectious disease followed by cardiovascular disease $(\mathrm{n}=10)$, gastrointestinal $(\mathrm{GI})(\mathrm{n}=9)$, neurology $(\mathrm{n}=5)$, orthopedics $(\mathrm{n}=4)$, and mental health $(n=2)$. Twenty one percent of the abstracts $(n=$ 27) were on subjects such as emergency room use or hospitalization that did not fit into a single clinical area. We classified these abstracts as "other".

We also stratified purpose of care by condition/specialty. Of the abstracts concerning diagnosis $(\mathrm{n}=19), 32 \%(\mathrm{n}=6)$ were related to cancer, including prostate, cervical, and thyroid cancers, followed by infectious disease $(21 \%, \mathrm{n}=4)$, cardiovascular disease and GI with $11 \%(\mathrm{n}=2)$ each, and orthopedics, mental health, and neurology (Fig. 4). Eleven percent $(\mathrm{n}=2)$ of diagnosis abstracts were classified as "other". Abstracts that described treatments or secondary prevention $(\mathrm{n}=91)$ focused on topics in pharmacy $(37 \%, \mathrm{n}=34)$, primarily inappropriate prescribing in the elderly, followed by other $(21 \%, \mathrm{n}=19)$, infectious diseases $(16 \%, \mathrm{n}=15)$, cardiovascular disease $(9 \%, \mathrm{n}=8)$, gastrointestinal $(8 \%, \mathrm{n}=7)$, cancer, neurology, orthopaedics, and mental health (Fig. 4). Screening/monitoring abstracts $(\mathrm{n}=16)$ were more narrowly distributed, with $63 \%$ (n $=10)$ related to cancer and $13 \%(\mathrm{n}=2)$ each for neurology, orthopedics, and other (Fig. 4).

\section{Discussion}

We conducted a search for studies of healthcare overuse literature and used the taxonomy we developed to review and classify the results. Prior reviews have looked at how overuse interacts with other variables, such as race/ethnicity [5] or highlighted specific 


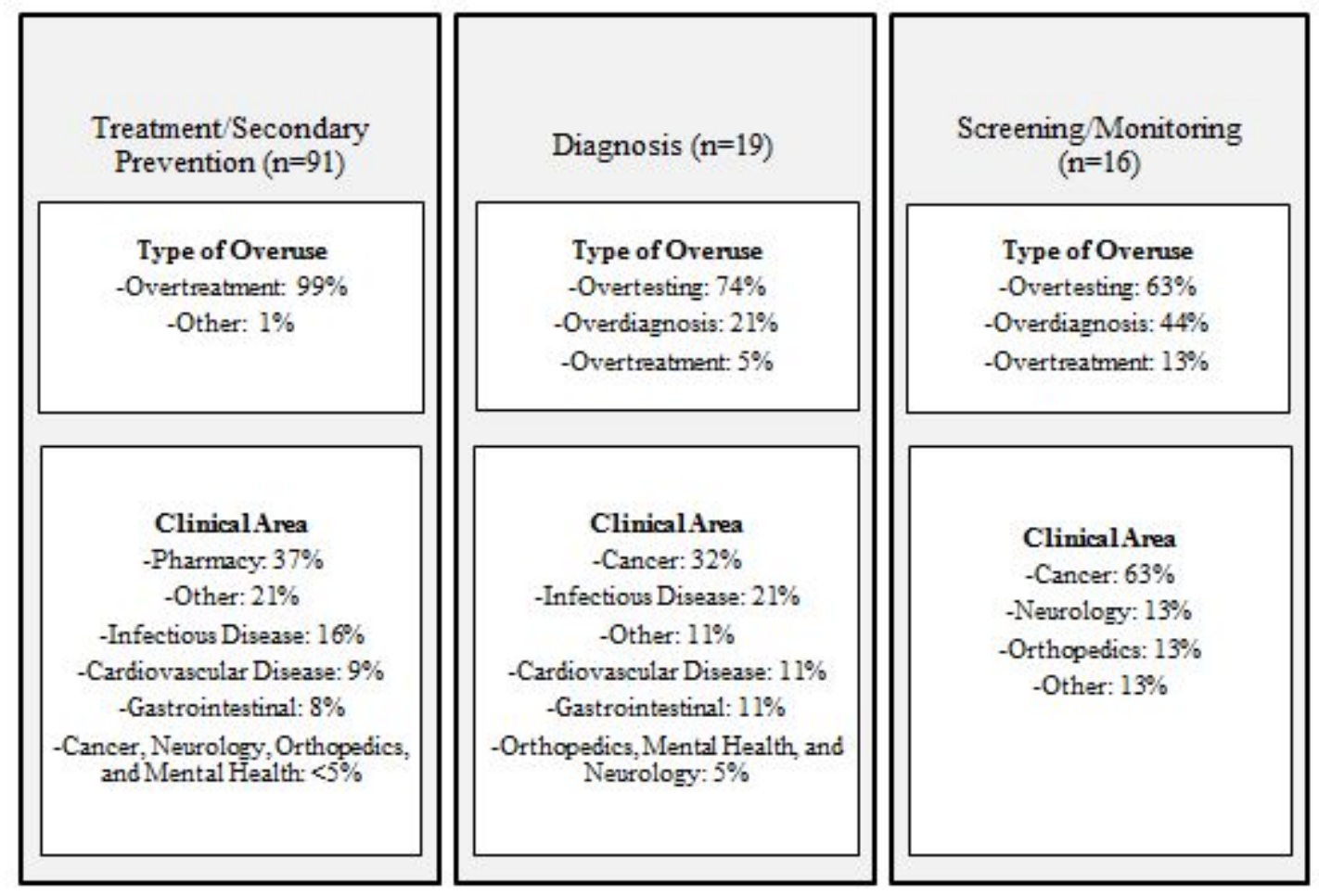

Fig. 4 Results of abstract review by purpose of care.

overuse topics [3]. Our goal was instead to provide an overview of the current state of all of the literature relating to overuse and to expand on the methods for categorization suggested by others [4], in order to create a taxonomy whose wide-spread adoption could have the potential to inform future research, practice and policy. We intended this as a guiding framework and initial proposal for further research to examine, categorize and develop strategies to reduce overuse overall and across specific clinical areas/specialties. We hope our study will stimulate clinicians, policy makers, and researchers to evaluate our proposed taxonomy and consider alternative or supporting methods.

The majority $(70 \%)$ of overuse research is observational, descriptive, and focuses on overtreatment (69\%) and overprescribing. There was only one controlled trial testing an intervention to reduce overuse [10]. This indicates that, while considerable description of overuse and speculation as to its causes and effects exists, little evidence to reduce overuse is actually being generated. The most commonly discussed driver of overuse was patient characteristics, yet the most common method to reduce overuse was guidelines/tools, which would not alter patient characteristics. Similarly, though education, or lack thereof, of a patient, provider or other participant was the least commonly mentioned driver of overuse, it was the third most frequently mentioned method to reduce overuse. This apparent disconnect between described drivers and suggested remedies may arise from measurement problems, lack of a conceptual framework for understanding causes of overuse, or a combination of these factors. The discussion of drivers, barriers, and methods was often speculative or relied primarily on observed associations. This pattern was also found by Powell et al. who noted that, "although these studies can improve the prediction of when overuse is more likely to occur, they do not specify why it occurs" or what to do about it [21]. More in-depth analysis is necessary to fully understand the factors driving overuse and to begin to craft effective solutions [21]. 
Only a small proportion of the literature (2\%) addressed primary prevention. In contrast, medication overuse, mainly inappropriate prescribing in the elderly, dominated our sample. Selection for these articles likely stemmed from authors' frequent use of the phrase "potentially inappropriate medications" (PIMs) clearly indicating healthcare misuse. "Potentially inappropriate medications" is a common term, used consistently to describe this phenomenon of overtreatment. Use of PIMs, especially in the elderly, has been broadly recognized as a prevalent problem associated with poor patient outcomes and high health care costs [22, 23]. As a result PIMs are now incorporated into quality measures and explicit criteria have been developed to improve physician prescribing [22]. This serves as an example of the steps that can be taken to identify and reduce overuse when its incidence is well understood and widely acknowledged.

Identifying overuse literature is difficult for many reasons, including that some terminology (e.g. guideline-discordant screening practices) describing overuse is unlikely to be captured by typical search terms. Available terms are nonspecific and the evidence-base encompasses many conditions, methodologies, and categorizations. This lack of search terms has been discussed as a persistent hindrance of overuse research [24]. Due to the number of results and the scope of our objective we only reviewed a random sample of abstracts instead of the full text of all identified articles. This limited the scope of our research as abstracts may not wholly capture the contents of the full article and a larger sampling frame might alter our findings. Nonetheless, information reported in abstracts likely reflects key study descriptors and findings and they are often the sole data source read.

We found that some examples of healthcare overuse are not labelled as such (e.g. guideline-discordant screening practices), which may prevent their identification in literature searches or studies of overuse. More widely agreed-upon phrases that indicate healthcare misuse, such as PIMs, would more readily identify that a study may have relevance to healthcare overuse. The formation and adoption of a common overuse vocabulary and taxonomy, such as the one proposed in this paper, would facilitate the identification of overuse literature and provide researchers with a common framework to categorize and label their work. Furthermore, this could facilitate the identification of high priority areas, drivers of overuse, barriers-to-reduce overuse, and effective and efficient implementation strategies. The Preventing Overdiagnosis and Lown Institute conferences are examples of scientific meetings that bring together researchers, clinicians, policy makers and patients to improve healthcare value by addressing and reducing overuse $[25,26]$. These venues could serve as useful forums to promote and disseminate research and practice related to healthcare overuse.

Increasing the accessibility of research that defines and discovers healthcare overuse will not only help clinicians reduce low-value care in practice but would identify gaps in research that may limit our ability to develop strategies to reduce overuse. Data on the prevalence and characteristics of healthcare overuse are crucial if policy makers are to prioritize and incentivize high-value care. One only needs to look at the example of PIMs to see that the instance of overuse most prevalent in the literature is also the case with established criteria for identifying overuse as well as organizational incentives and concerted efforts being made to reduce it.

\section{Conclusions}

In conclusion, a large and varied body of literature on overuse exists but is difficult to identify. Our taxonomy classified research into three categories, (purpose of care, type of overuse, and state-of-the-research) and was able to effectively organize the existing literature and may help efficiently focus research and clinical efforts into high priority areas. The majority of literature is observational and 
evaluates overtreatment; mainly inappropriate prescribing in the elderly. There is scant literature on overuse in primary prevention or barriers-to-reducing overuse. Future overuse research is needed in diagnostic care and non-drug treatments, as well as controlled trials of interventions to reduce overuse. A common taxonomy, such as the one we developed, could help researchers categorize their work, assist clinicians and policymakers in identifying and implementing findings, and guide future research to improve health care quality.

\section{Acknowledgments}

The authors would like to thank Rod MacDonald ${ }^{1}$, MS for his contributions to the design of the taxonomy and Brent Taylor ${ }^{1}$, PhD, MPH for his contributions to the design of the taxonomy and advice on data presentation for which compensation was not received.

${ }^{1}$ Center for Chronic Disease Outcomes Research and the Minneapolis VA High Value Care Initiative, Minneapolis VA Health Care System, Minneapolis, MN 55417, USA

\section{References}

[1] Welch, H. G., Schwartz, L. M., and Woloshin, S. 2011. Overdiagnosed: Making People Sick in the Pursuit of Health. Boston: Beacon Press.

[2] Delaune, J., and Everett, W. 2008. Waste and Inefficiency in the US Health care System-Clinical Care: A Comprehensive Analysis in Support of System-wide Improvements. Cambridge, MA: New England Healthcare Institute.

[3] Morgan, D., Wright, S., and Dhruva, S. 2015. "Update on Medical Overuse.” JAMA Intern Med. 175: 120-4.

[4] Korenstein, D., Falk, R., Howell, E., Bishop, T., and Keyhani, S. 2012. "Overuse of Health Care Services in the United States." Archives of Internal Medicine 172: 171-8.

[5] Kressin, N., and Groeneveld, P. 2015. "Race/Ethnicity and Overuse of Care: a Systematic Review." The Milbank Quarterly 93: 112-38.

[6] "Primary Prevention, Screening, Monitoring." Miller-Keane Encyclopedia \& Dictionary of Medicine, Nursing \& Allied Health—Revised Reprint, 7th Edition. 7th ed. Philadelphia: W.B. Saunders. http://medical-dictionary.thefreedictionary.com/ 2003.
[7] "Diagnosis." The American Heritage Medical Dictionary. Boston: Houghton Mifflin Co. http://medical-dictionary.thefreedictionary.com/ 2007.

[8] “Treatment, Screening, Monitoring." Mosby's Medical Dictionary. 8th ed. St. Louis: Elsevier Health Sciences. http://medical-dictionary.thefreedictionary.com/ 2009.

[9] Greenberg, J., and Green, J. 2014. "Over-testing: Why More is not Better." The American Journal of Medicine 127 (5): 362-3.

[10] Tannenbaum, C., Martin, P., Tamblyn, R., Benedetti, A., and Ahmed, S. 2014. "Reduction of Inappropriate Benzodiazepine Prescriptions among Older Adults through Direct Patient Education: the EMPOWER Cluster Randomized Trial." JAMA Internal Medicine 174 (6): 890-8.

[11] Janzing, H., and Broos, P. 2001. "Routine Monitoring of Compartment Pressure in Patients with Tibial Fractures: Beware of Overtreatment!' Injury 32 (5): 415-21.

[12] Kim, S., Park, W., Lee, K., Kang, C., Bang, J., Kim, H., Kim, E., Oh, M., and Choe, K. 2004. "Outcome of Inappropriate Initial Antimicrobial Treatment in Patients with Methicillin-resistant Staphylococcus Aureus Bacteraemia." Journal of Antimicrobial Chemotherapy 54 (2): 489-97.

[13] Mann, E., Haastert, B., Bohmdorfer, B., Fruhwald, T., Iglseder, B., Roller-Wirnsberger, R., and Meyer, G. 2013. "Prevalence and Associations of Potentially Inappropriate Prescriptions in Austrian Nursing Home Residents: Secondary Analysis of a Cross-sectional Study." Wiener Klinische Wochenschrift 125 (7-8): 180-8.

[14] Manolakaki, D., Velmahos, G., Spaniolas, K., de Moya, M., and Alam, H. 2009. "Early Magnetic Resonance Imaging is Unnecessary in Patients with Traumatic Brain Injury." Trauma 66 (4): 1008-12.

[15] Tan, A., Kuo, Y., Elting, L., and Goodwin, J. 2013. "Refining Physician Quality Indicators for Screening Mammography in Older Women: Distinguishing Appropriate Use from Overuse." Journal of the American Geriatrics Society 61 (3): 380-7.

[16] Nistico, J., Hage, J., Schoch, P., and Cunha, B. 2013. "Unnecessary Repeat Clostridium Difficile PCR Testing in Hospitalized Adults with C. Difficile-negative Diarrhea." European Journal of Clinical Microbiology and Infectious Diseases 32 (1): 97-9.

[17] Njuguna, J., Muita, J., and Mundia, G. 2009. "Malaria Morbidity and Temperature Variation in a Low Risk Kenyan District: a Case of Overdiagnosis?” International Journal of Biometeorology 53 (3): 299-304.

[18] Mitchell, P. 2012. "Bipolar Disorder: the Shift to Overdiagnosis.” Canadian Journal of Psychiatry 57 (11): 659-65.

[19] Rigler, S., Jachna, C., Perera, S., Shireman, T., and Eng, 
M. 2005. "Patterns of Potentially Inappropriate Medication Use across Three Cohorts of Older Medicaid Recipients." Annals of Pharmacotherapy 39 (7-8): 1175-81.

[20] Ye, S., Rabbani, L., Kelly, C., Kelly, M., Lewis, M., Paz, Y., Peck, C., Rao, S., Bokhari, S., Weiner, S., and Einstein, A. 2015. "Can Physicians Identify Inappropriate Nuclear Stress Tests? An Examination of Inter-rater Reliability for the 2009 Appropriate Use Criteria for Radionuclide Imaging." Circulation: Cardiovascular Quality and Outcomes 8 (1): 23-9.

[21] Powell, A., Bloomfield, H., Burgess, D., Wilt, T., and Partin, M. 2013. "A Conceptural Framework for Understanding and Reducing Overuse by Primary Care Providers." Medical Care Research and Review 70 (5): 451-72.

[22] Campanelli, C. 2012. “American Geriatrics Society Updated Beers Criteria for Potentially Inappropriate
Medication Use in Older Adults: the American Geriatrics Society 2012 Beers Criteria Update Expert Panel." Journal of the American Geriatric Society 60 (4): 616-31.

[23] Fu, A., Jiang, J., Reeves, J., Fincham, J., Liu, G., and Perri, M. 2007. "Potentially Inappropriate Medication Use and Healthcare Expenditures in the US Community-dwelling Elderly." Medical Care 45: 472-6.

[24] Morgan, D., Brownlee, S., Leppin, A., Kressin, N., Dhruva, S., Levin, L., Landon, B., Zezza, M., Schmidt, H., Saini, V., and Elshaug, A. 2015. "Setting a Research Agenda for Medical Overuse.” British Medical Journal 351: 4534.

[25] Preventing Overdiagnosis [Internet]. [Place unkown]: [Publisher unknown]; [date unkown] [cited 2015 Dec 21] Available from:http://www.preventingoverdiagnosis.net/.

[26] Lown Institute [Internet]. Brookline, MA: The Lown Institute; [date unkown] [cited 2015 Dec 21] Available from: http://conference.lowninstitute.org/. 